Sektion 9

\title{
Pädiatrische Pneumologie
}

Die Sektionssitzung der Sektion 9 war traditionsgemäß schlecht besucht. Die meisten pädiatrischen Pneumologinnen und Pneumologen, die auf dem Kongress anwesend waren, sind keine DGP-Mitglieder. Da eine DGP-Mitgliedschaft automatisch zum Verlust einer Reisekostenübernahme trotz eingeladenem Vortrag oder Vorsitz führt, wird sich dies in Zukunft aller Voraussicht nach auch nicht wesentlich ändern.

Als erstes wurden die Aktivitäten auf dem aktuellen Jahreskongress besprochen und bewertet. Sehr positiv ist die Anzahl an interessierten Zuhörerinnen und Zuhörern bei dem PG-Kurs („CF-Basics für Pneumologen“) und den verschiedenen Symposien unter Beteiligung der Sektion 9 aufgefallen. Auch in den vielen Diskussionsbeiträgen konnte man erkennen, dass die Kinderpneumologie an sich, deren Vernetzung mit Erkrankungen des Erwachsenenalters und auch die Mukoviszidose zunehmend an Beachtung zu gewinnen scheinen.

Außerdem wurde ausgesprochen positiv über die zunehmende Kooperation der beiden Fachgesellschaften DGP und GPP diskutiert. Die Verwaltung der Mitgliederdatei der GPP in den Geschäftsräumen der DGP in Berlin kommt ausgesprochen gut voran, und ein entsprechender Kooperationsvertrag liegt nun in der finalen Fassung zur Unterschrift bereit. Dies ist aus Sicht der Kinderpneumologie ein hocherfreulicher Schritt.

Letzter Punkt war die Planung der Beiträge auf dem Jahreskongress der DGP 2018 in Dresden. Verschiedene Vorschläge wurden entwickelt, sodass zeitnah entsprechende Anträge bei der Programmkommission eingehen werden.

Prof. Philippe Stock, Hamburg 